\title{
Global Citation Impact rather than Citation Count
}

\author{
Gohar Rehman Chughtai, Jia Lee, \\ Muhammad Mehran Arshad khan \\ College of Computer Science, Chongqing University, \\ Chongqing 400044, China \\ Rashid Abbasi \\ College of Computer Science and Technology, \\ Anhui University, 230601, \\ Hefei, China
}

\author{
Asif Kabir \\ College of Communication Engineering, Chongqing \\ University, 400044, China
}

Muhammad Arshad Shehzad Hassan

State Key Laboratory of Power Transmission Equipment \& System Security and New Technology, School of Electrical Engineering, Chongqing University, Chongqing 400044, China

\begin{abstract}
The progressing bloom in the tome of scientific literature available today debars researchers from efficiently shrewd the relevant from irrelevant content. Researchers are persistently engrossed in impactful papers, authors, and venues in their respective fields. Impact of an article depends on the citation received but just a citation count can't give readers indepth information about the article. That is the reason some articles are quantified unfairly on the basis of a citation count. In this paper, Global Citation Impact (GCI) is proposed which addresses the issue of considering citations of papers equally. Intuitively, the papers citing a paper are not of the same worth. The proposed index not only considers the number of citations (popularity) like many existing methods did but also considers the worth of citations (prestige). Results and discussions show that researcher whose work is cited by other prestigious papers gets higher rank which is quite fair crediting for research impact.
\end{abstract}

Keywords-Citation weighting; popular; global citations; prestigious; Global Citation Impact (GCI); research impact

\section{INTRODUCTION}

Currently, scientific work evaluation and quantification is an important research area for researchers, for the sake of unbiased and fair crediting of their research work. It is a challenging task for an index to be the best fit and acceptable to the whole scientific community. Scientific work evaluation is necessary to decide whether or not a researcher is promoted, is suitable as a principal investigator for a project, should get a $\mathrm{PhD}$ degree, should be given tenure or should be awarded an important research funding.

Traditionally, research work contribution crediting is done by involving number of papers and number of citations by the state of the art H-Index [1], G-Index [2] q2-index [3] and all the variant of $\mathrm{H}$-index such as A-index [4], R-index and ARindex [5], w-index a significant improvement of H-index [6], fractional counting of authorship [7], Weighted Citation [8] and E-index [9], [10]. Reviews various indicators that can possibly be used to measure the performance of an author. These variations of $\mathrm{H}$-index have considered the citations as their target to quantify the scientific work, but they punish the new emerging scientists because of lesser citations received. To address this issue career length is considered in the quantification of research to address the ignorance of new scientists in the field by m-quotient [4]. Later, co-authorship contributions [11], such as the order of authors contributing to paper and a number of authors in a paper is considered by Kth-rank [12] and fractional H-index. The self-citations should not be given the same weight as citations by others issue is investigated and F-Index [13] was proposed. Finding the rising star in academia [14] where the star is the authors who have not enough citation at the start of their career but predicted as a rising star in the future. Their contribution actually highlights the new researchers irrespective of traditional indexing methods to give credits to researchers on the basis of citations and number of publications. Topic-based ranking of authors [15] and consistent annual citation based index [16], identifying authorities of a given topic within a particular domain [17] which is a great contribution to expert finding. Impact of hot paper on individual's research contribution demonstrated by [18] which is an encouragement for new emerging researchers. The IF of a journal, therefore, is not representative of the number of citations of an individual article in the journal [19].

Existing indexing methods have majorly considered the number of citations (popularity), researcher age factor, the order of authors (co-author contribution) and self-citations, while prestige (worth of citing papers) was ignored. The difference between popularity and prestige is provided in the following example. Suppose a researcher has two papers A and $B$ and both are cited by 10 papers. Eight of the papers that cited paper A are written by the prestigious researchers while only three of the papers cited paper B are written by prestigious researchers. Though the number of citations is the same for both paper A and B, it seems that paper A is more useful for researcher $A$, due to getting the attention of eight prestigious researchers. In this work, considering the worth of papers citing a paper is referred to as the impact of global citations. Global Citation Index (GC-Index) is proposed which considers the worth of citing papers for indexing researchers. The more the worth of the citing papers of a paper the more the prestige it has and is highly important for the scientific community.

The major contributions of this paper towards researcher indexing are (1) differentiating between popularity (citation count) and global impact of a paper (2) highlighting positive 
impact of prestigious paper citing a paper and (3) proposal of PageRank based method to calculate the global impact of a paper which shows significance of paper. To the best of our knowledge, this work will give more insight into the significance of an article which could be helpful for readers to find the quality article.

\section{Global Citation ImPACt (GCI)}

The popularity of an article depends on the number of citation received by an article without knowing the worth of citing the paper. In past publication count and citation count is extensively used in author ranking in most of the bibliometric indicators, but this way we cannot find the worth of the article if we don't know the citing article and author. H-index is generally acknowledged by major online databases, albeit, one blemish of $\mathrm{H}$-index is that its value never goes down over time.

Scientific impact measurement achieves a move from popularity to the prestige of scientific productions. Since PageRank is acquainted with the scholastic assessment. In spite of these researchers have advanced the improvement of scholarly impact assessment, the most eminent constraint of the PageRank-based assessment tool is estimating the prestige of citation-publication network. The PageRank algorithm and its variants were used for the assessment of various types of citation-publication networks. The question has been raised whether better assessment results were depended directly on an author network or on a publication-citation network [20].

PageRank calculation without anyone else is initially intended to use web pages ranking, web pages propagations are considered as equivalent significance, accentuating the restrictive measurement. Specifically using PageRank to assess the academic impact regards all citations as equivalent weights, which adequately ignores the impact from citing authors. HR-PageRank, incorporate weighted PageRank according to individual's H-index, and pertinence between citing and cited papers [21]. We contend that the PageRankindex is an impartial and more nuanced metric to evaluate the publication records of researchers contrasted with existing measures [22].

Basically, a global citation impact is the weighted impact of the citation on the basis of the global weight of its citing article which shows how worthy article is, not just popularity. In other words, if a paper is cited by impactful paper then it is more worthy because it attracts a more impactful audience. In this paper, the impact of global citations is considered. It is quite reasonable to think that a paper cited by many prestigious papers is likely to be more worthy as compared to a paper cited by not prestigious papers. Fig. 1 provides an example of four nodes where vertices represent papers and edges represent citations. It demonstrates that how the global citation impact is effective in the provision of more insight into paper significance.

A directed paper-citation graph in Fig. 1 is a pair $(V ; E)$, where edges point toward and away from vertices. For a vertex $v_{i} \in V ; \operatorname{In}\left(v_{i}\right)$ is the set of vertices that point to it and $\operatorname{Out}\left(v_{j}\right)$ is the set of vertices that $v_{i}$ point to, such that:

$\operatorname{In}\left(v_{i}\right)=\left\{v_{j} \in V\left(v_{j}, v_{i}\right) \in \varepsilon\right\}$

$\operatorname{Out}\left(v_{j}\right)=\left\{v_{j} \in V\left(v_{i}, v_{j}\right) \in \varepsilon\right\}$

$V\left(v_{i}\right)=\operatorname{In}\left(v_{i}\right) \cup \operatorname{Out}\left(v_{i}\right)$

In Table I, rows show the in-degree of nodes while columns show the out-degree of nodes calculating prestige of paper using PageRank.

In Table II, P1 got the highest global weight because it is cited by P2 which has the highest local weight and P4. Every citation has different weight then it should be considered in the researcher indexing. The local weight is calculated by using PageRank's (4) and global weight is the summation of the paper's local weights citing a paper.

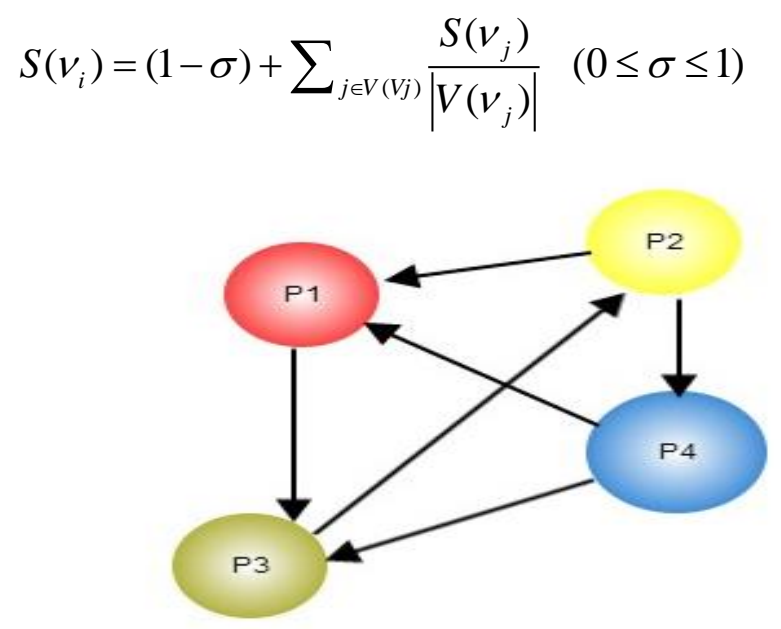

Fig. 1. Paper-citation graph.

TABLE I. MATRIX OF DEGREES OF NODES

\begin{tabular}{|l|l|l|l|l|}
\hline Paper & P1 & P2 & P3 & P4 \\
\hline P1 & 0 & $1 / 2$ & 0 & $1 / 2$ \\
\hline P2 & 0 & 0 & 1 & 0 \\
\hline P3 & 1 & 0 & 0 & $1 / 2$ \\
\hline P4 & 0 & $1 / 2$ & 0 & 0 \\
\hline
\end{tabular}

TABLE II. GLOBAL AND LOCAL WEIGHT

\begin{tabular}{|l|l|l|l|l|l|l|}
\hline Paper & Local weight & Global weight & $\boldsymbol{W}_{\text {cit }} 1$ & $\boldsymbol{W}_{\text {cit }} 2$ & $\boldsymbol{W}_{\text {cit }} 3$ & $\boldsymbol{W}_{\text {cit }} 4$ \\
\hline P1 & 1.25 & 3.25 & 0 & 2.50 & 0 & 0.75 \\
\hline P2 & 2.50 & 2 & 0 & 0 & 2 & 0 \\
\hline P3 & 2 & 2 & 1.25 & 0 & 0 & 0.75 \\
\hline P4 & 0.75 & 2.50 & 0 & 2.50 & 0 & 0 \\
\hline
\end{tabular}


$S\left(v_{i}\right)$ and $S\left(v_{j}\right)$ denote the score of vertex $v_{i}$ and $v_{j}$ respectively, $V\left(v_{i}\right)$ and $V\left(v_{j}\right)$ denote the set of vertices connecting with $v_{i}$ and $v_{j}$ respectively, and $\sigma$ is a damping factor that integrates into the computation the probability of jumping from a given vertex to another random vertex in the graph.

Originally, Google PageRank algorithm assumed the parameter $\mathrm{d}$ to be 0.15 . This value was incited by the informal observation that a person surfing the web will usually follow the order of 6 hyperlinks, equivalent to a leakage probability $\mathrm{d}$ $=1 / 6 \simeq 0.15$, before becoming either bored or frustrated with this line of search and begin a new search. In the context of citations, it is common hypothesis that entries in the reference list of a typical paper are collected following somewhat shorter paths of average length 2 , making the choice $d=0.5$ more appropriate for a similar algorithm applied to the citation network. is that approximately $50 \%$ of the articles [23].

In our scenario, $\mathrm{GCI}$ is computed as

$W_{\text {local }}(n)=\sigma\left(\frac{1}{G}\right)+(1-\sigma) \sum_{m \in L(n)} \frac{W_{\text {local }}(m)}{C(m)}$

Where $G$ is the total number of nodes (papers) in the graph, $\sigma$ is the random damping factor $(n)$ is the set of papers that link (cite) $n$ and $C(n)$ is the out-degree of the node $m$.

Therefore,

Global citation weight of the citation is as follow:

$W_{\text {Global }}(n)=\sum_{m \in L(n)} W_{\text {local }}$

Whereas $W_{\text {Global }}(n)$ is the global weight of the node (paper). To index the author on the basis of global citation weight as H-index does use citation count we have arranged the documents in descending order on the basis of global citation weight. The index which satisfies, $r_{0}(n) \leq W_{\text {Global }}(n)$ , where $r_{0}$ is the highest rank, will be the GCI-rank of an author.

\section{EXPERIMENTS}

Authors have carried out a series of experiments which is discussed in detail in this section in which analysis for the changes in the ranks of researchers is provided on the large real bibliographic dataset.

\section{A. Dataset}

Initially, we have taken 1000 researchers from Cite seer then completed their graph. In this process of getting the citing papers of each paper of a researcher, a total number of authors becomes 24567 and the total number of publications is 140883. Our data set consists of data variables PID (Publication ID), Authors, in links, out links and citations. Authors have removed inconsistencies and duplications in the dataset in order to get accurate results and completed the data set by extracting each publication of an author, its citing publications and completed the matrix of the dataset. Authors have chosen 30 authors having a difference in their rank from $50,40,30,20,10$ ranges of $\mathrm{H}$-index rank so that to analyze the variation with respect to proposed GCI-Impact ranks.

Fig. 2 represents the paper-citation graph and the process of selection of top authors for analysis. In graph red nodes represents the papers of an author and blue lines are the edges which represent the in degree and the out-degree of an article. In the following Table III, authors have given some statistics of data set used for simulations.

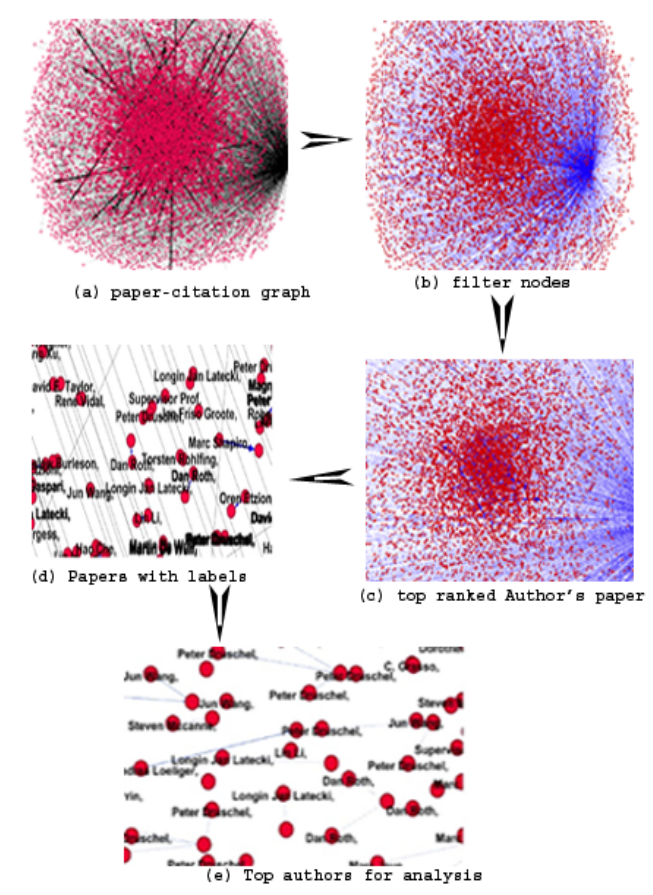

Fig. 2. Paper-citation Directed Graph.

TABLE III. CITE SEER DATASET

\begin{tabular}{|l|l|l|l|}
\hline $\begin{array}{l}\text { No. of } \\
\text { Authors }\end{array}$ & $\begin{array}{l}\text { No. of } \\
\text { publication }\end{array}$ & $\begin{array}{l}\text { Average No. of } \\
\text { Citation }\end{array}$ & $\begin{array}{l}\text { Average No. of Global } \\
\text { Citation }\end{array}$ \\
\hline 24567 & 140883 & 9.80 & 55.11 \\
\hline
\end{tabular}

\section{B. Parameter Settings}

In $\mathrm{H}$-index square root of total citations of all publications of an author are divided by Proportionality constant " $a$ " whose values range from 3 to 5 . Authors have set the value of " $a n$ " equal to 4 because it's a minimum number of publications and number of citations per paper per year of an author. Higher the value of " $a$ " higher is the consideration of highly cited publications into $\mathrm{H}$-index core which ignore the real participating articles in the $h$ core. 
Authors have tested GC-Index on two different values of "damping factor", 0.85 which is used in PageRank calculation and 0.50 to represent paper citation network according to [23]. They have shown that average citation link in the citation network for an academic researcher is $1 / 2$ that's why 0.50 suits more for paper citation network.

\section{Results and Discussions}

The results of the proposed GCI are compared with $\mathrm{H}$ Index. Authors have chosen 30 researchers randomly for our analysis. Table IV shows papers, citations, the rank of authors according to H-Index and GCI-rank, average citations, average global citations, and variation in the rank. Average $G_{\text {cit }}$ shows that how much the citing paper is important and how much it serves the audience? If a paper attracts well- known researchers then it definitely means that work has more worth. Knowing that how much worth the citing articles have is very important to analyze the quality of one's research. GCIndex shows the variation in rank along with variation in damping factor on 0.50 and 0.85 , which also affect the results.

1) Comparison of H-Index and GCI-rank with damping factor 0.85

Table IV has shown the impact of global citations on author rank with damping factor 0.85 . Their scenarios are discussed, that is; position earned, a position lost and position stable by authors with respect to H-Index. In Table IV, variation in rank column + symbol means position earned, symbol means position lost and 0 means position stable.

TABLE IV. COMPARISON OF RANKS ON DAMPING FACTOR 0.85

\begin{tabular}{|c|c|c|c|c|c|c|c|c|c|c|}
\hline S No & Authors & Publications & Citations & $\begin{array}{r}\text { Average } \\
\text { cit }\end{array}$ & $\begin{array}{r}\text { Average } \\
G_{c i t}\end{array}$ & H-Index & GC-Index & H-rank & GC-rank & $\begin{array}{l}\text { Variation } \\
\text { in rank }\end{array}$ \\
\hline 1 & Peter Druschel & 124 & 10185 & 82.137 & 1374.121 & 50 & 52 & 1 & 1 & 0 \\
\hline 2 & Oren Etzioni & 128 & 5516 & 43.093 & 622.125 & 44 & 30 & 2 & 4 & +2 \\
\hline 3 & Dan Roth & 157 & 4022 & 25.617 & 438.051 & 35 & 32 & 3 & 3 & 0 \\
\hline 4 & Lin $\mathrm{Li}$ & 292 & 4661 & 15.962 & 178.537 & 32 & 21 & 4 & 7 & -3 \\
\hline 5 & Steven Mccanne & 53 & 4248 & 80.150 & 1689.264 & 30 & 40 & 5 & 2 & +3 \\
\hline 6 & Jun Wang & 268 & 3872 & 14.447 & 152.947 & 26 & 15 & 6 & 10 & -4 \\
\hline 7 & Judea Pearl & 92 & 2048 & 22.260 & 353.434 & 25 & 27 & 7 & 5 & +2 \\
\hline 8 & Hao Che & 160 & 2353 & 14.706 & 126.618 & 24 & 14 & 8 & 11 & -3 \\
\hline 9 & Marc Shapiro & 69 & 1775 & 25.724 & 365.144 & 22 & 19 & 9 & 8 & +1 \\
\hline 10 & Eyal Amir & 60 & 1173 & 19.55 & 299.733 & 21 & 15 & 10 & 10 & 0 \\
\hline 11 & Ira Cohen & 57 & 1252 & 21.964 & 262.368 & 21 & 13 & 10 & 12 & -2 \\
\hline 12 & Giedrius Slivinskas & 60 & 1265 & 21.083 & 158.216 & 20 & 11 & 11 & 14 & -3 \\
\hline 13 & Robert Nieuwenhuis & 59 & 1058 & 17.932 & 259.186 & 19 & 14 & 12 & 11 & +1 \\
\hline 14 & Dorothea Wagner & 110 & 1140 & 10.363 & 108.863 & 19 & 11 & 12 & 14 & -2 \\
\hline 15 & Longin Jan Latecki & 86 & 1188 & 13.813 & 169.976 & 19 & 19 & 12 & 8 & +4 \\
\hline 16 & Jan Friso Groote & 75 & 1006 & 13.413 & 117.906 & 19 & 16 & 12 & 9 & +3 \\
\hline 17 & Marc Joye & 77 & 915 & 11.883 & 101.103 & 17 & 16 & 13 & 16 & -3 \\
\hline 18 & Shigang Chen & 45 & 1223 & 27.177 & 320.755 & 17 & 13 & 13 & 12 & +1 \\
\hline 19 & Kristian Torp & 46 & 923 & 20.065 & 142.782 & 16 & 11 & 14 & 14 & 0 \\
\hline 20 & S. Keshav & 38 & 805 & 21.184 & 254.263 & 16 & 12 & 14 & 13 & +1 \\
\hline 21 & Jian Chen & 83 & 831 & 10.012 & 91.831 & 16 & 14 & 14 & 11 & +3 \\
\hline 22 & Yang Yu & 53 & 688 & 12.981 & 111.207 & 15 & 12 & 15 & 13 & +2 \\
\hline 23 & Judith Donath & 39 & 554 & 14.205 & 161.743 & 14 & 11 & 16 & 14 & +2 \\
\hline 24 & Wei Sun & 76 & 588 & 7.7368 & 95.210 & 13 & 7 & 17 & 16 & +1 \\
\hline 25 & Michal Feldman & 25 & 608 & 24.32 & 273.52 & 13 & 11 & 17 & 14 & +3 \\
\hline 26 & Hans-rea Loeliger & 56 & 1920 & 34.285 & 588.75 & 12 & 23 & 18 & 6 & +12 \\
\hline 27 & Y. Rich & 21 & 553 & 26.333 & 331.047 & 12 & 10 & 18 & 15 & +3 \\
\hline
\end{tabular}




\begin{tabular}{|l|l|l|l|l|l|l|l|c|c|c|}
\hline 28 & Abhishek Kumar & 12 & 322 & 26.833 & 392 & 10 & 7 & 19 & 16 & +3 \\
\hline 29 & A. Bondavalli & 22 & 173 & 7.863 & 67.090 & 7 & 7 & 20 & 16 & +4 \\
\hline 30 & Gerrit Muller & 24 & 66 & 2.75 & 12.333 & 4 & 2 & 21 & 17 & +4 \\
\hline
\end{tabular}

Authors have lost their rank in CGH-index even though

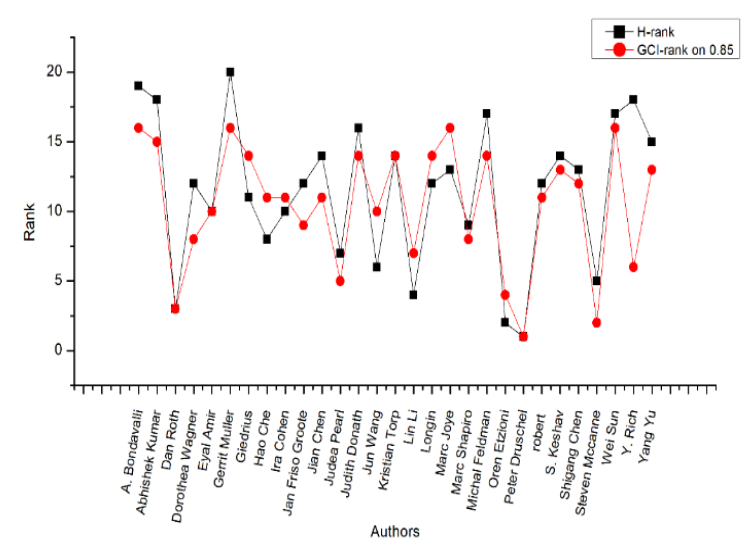

Fig. 3. Comparison of H-index and CGH with damping factor 0.85 .

2) Scenario 1: Relocation with respect to H-Index: position up

Fig. 3 depicts the variation of ranks of researchers on damping factor 0.85 and more detailed elaboration of their results is explained in Table IV Judea Pearl who's H-Index was 25 and rank at position 7, with GC-Index his rank is increased by 2 . This is because of his two publications having citations from worthy nodes which enhanced its rank. Another case in which the author gets a higher rank is the highest variation in selected authors for analysis. Hans-rea Loeliger having 56 and $\mathrm{H}$-index is 12 but according to our proposed GC-Index, its index increased to 23. His index increased by 11 because its average global citation is quite higher, which is 588.75. He should be given higher rank because his work stimulated many other worthy papers in that domain. S. Keshav and Judith Donath, have almost same number of publications and their H-Index is 16 and 14, respectively. S. Keshav has average global citations 254.263 and Judith Donath has average global citations 161.743 on the basis of this S. Keshav has more worthy citations which so he should have a higher rank than Judith Donath. With respect to our proposed GC-Index, S. Keshav earned 7 positions higher than in H-Index rank while Judith Donath got just 2 positions high in with GC-Index.

\section{3) Scenario 2: Relocation with respect to $H$-index rank:} position down

Some authors have enough citations of their publications and they have higher $\mathrm{h}$ index but beside citations, according to their global weight, these citations are not so important because these are not weighted heavily enough to include it into its effective rank calculation. That's why these authors have lost their position with respect to $\mathrm{CGH}$-index. As in $\mathrm{h}$ index, every citation of a publication matters a lot. In case of $\mathrm{CGH}$-index not only the citation is considered but it must be an important publication to be the part of $\mathrm{CGH}$-index core. some of them have a higher average global citation. It is just because they have few publications which have a more weighted citation but few are not so important that could include them in the $\mathrm{CGH}$-index core. On average they have a high global citation but when computing CGH-index individual publication should have more weighted citations which can include them in CGH core. As in Table $\mathrm{V}$ two authors Ira chohen and GiedriusSlivinskas have almost an equal number of publication 57 and 60 and 1252, 1265 citations respectively. Both have lost the rank in CGH-index. Irachohen whose $\mathrm{h}$ index is 21 and rank 10 he lost rank by 2 and index by 8 while GiedriusSlivinskas has,h index 20 and rank 11 has lost his $\mathrm{h}$ rank by 3 and CGH-index by 9 . GiedriusSlivinskas has lost more than Irachohen even though he has more publication and citation but he has average global citation 158.216lesser than average global citation of Irachohen whose average global citation is 262.368 which effects its rank and index more than Irachohen. Oren Etzioni has the highest global citation weight in Table VI that's 622.125 but still, he lost his rank by 2 because it is replaced by Steven Mccanne, whose $G_{c i t}$ is 1689.264 which is high enough than Etzioni which ranked him by 3 .

4) Scenario 3: Position Stable With Respect to H-index

Peter Druschel has H-index 50 and his CGH-index is 52 which was already at first rank in $\mathrm{h}$ index rank other authors in Table VII has lost CGH-index but their rank remains stable. Damping factor is also important in graph-based ranking as we varied the damping factor rank of authors is also a varied little bit.

5) Comparison of H-Index and GC-Index on damping factor 0.50

Authors have discussed the comparison of CGH-index in following three different scenarios. Following scenarios clearly, describe the variation in results by varying the damping factor.

As in Table V first author, Steven Mccanne got higher rank which increases by 3 and he replaced Oren Etzioni because Steven has 53 publication 4248 citations and 1689.264 average global citation count while Etzioni has 128publication 5516 citation and 622.125 global citation count. Although he has more publications and citations, his global citation count is very less than Steven that's why he has replaced him and got position 2. In another case author Hansrea Loeliger whose publication is 56, citations are 1920 and average global citation count is 588.75 earned position by 13 . No other author has a high average global citation but they have a higher citation which gives them higher rank in $\mathrm{H}$ index. Fig. 5 shows the gain in rank with respect to $\mathrm{CGH}-$ index. 


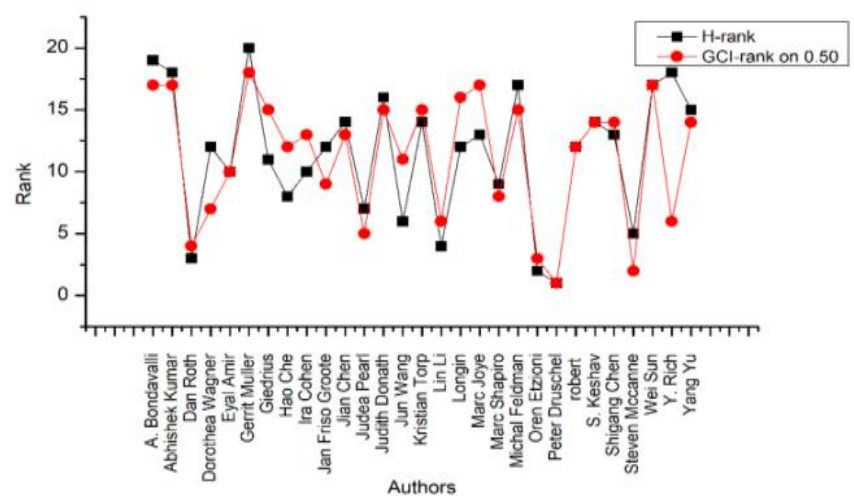

Fig. 4. Comparison of H-Index and CGH with damping factor 0.50.
Fig. 4 shows the variation of ranks among different authors on damping factor 0.50 which are explained in Table VI, first author Oren Etzioni has lost rank by 1 and he has been replacing by Steven whose average global citation count is quite higher than Oren which is 1689.264 and Oren has just 622.125. Dan Roth has,h-index rank 3 but here he lost rank by 1 and replaced by Oren Etzioni whose average global citation count is 622.125 while Dan Roth has 438.051 which is lesser than him that's why he could not maintain his rank.

In Table VII first author Peter Druschel has maintained his position on H-index rank on both damping factor values because he has highest average Global citations. Some of the authors have lost their $\mathrm{CGH}$-index but when ranked according to it, they have maintained their position with respect to $\mathrm{H}$ index because variations occur in other's rank.

TABLE V. SCENARIO 1 "Relocation with Respect CGH-INDEX RANK: Position UP"

\begin{tabular}{|l|l|l|l|l|l|l|}
\hline S No & Authors & publications & citations & Average $\boldsymbol{G}_{\boldsymbol{c i t}}$ & H-index rank & Earned Position on 0.50 \\
\hline 1 & Steven Mccanne & 53 & 4248 & 1689.264 & 5 & +3 \\
\hline 2 & Judea Pearl & 92 & 2048 & 353.434 & 7 & +2 \\
\hline 3 & Longin Jan Latecki & 86 & 1188 & 169.976 & 12 & +3 \\
\hline 4 & Hans-rea Loeliger & 56 & 1920 & 588.75 & 18 & +13 \\
\hline 5 & Robert Nieuwenhuis & 59 & 1058 & 259.186 & 12 & +1 \\
\hline 6 & Kristian Torp & 46 & 923 & 142.782 & 9 & +1 \\
\hline 7 & Marc Shapiro & 69 & 1775 & 365.144 & 16 & +3 \\
\hline 8 & Judith Donath & 39 & 554 & 161.743 & 17 & +2 \\
\hline 9 & Michal Feldman & 25 & 608 & 273.52 & 18 & +2 \\
\hline 10 & Y. Rich & 21 & 553 & 331.047 & 19 & +2 \\
\hline 11 & Abhishek Kumar & 12 & 322 & 392 & 20 & +1 \\
\hline 12 & A. Bondavalli & 22 & 173 & 67.090 & 21 & +3 \\
\hline 13 & Gerrit Muller & 24 & 66 & 12.333 & +2 \\
\hline
\end{tabular}

TABLE VI. “SCENARIO 2: RELOCATION WITH RESPECT TO CGH-INDEX RANK ON 0.50: POSITION DOWN”

\begin{tabular}{|c|c|c|c|c|c|}
\hline Authors & Publications & Citations & Average $G_{c i t}$ & H-index rank & Position Lost \\
\hline Oren Etzioni & 128 & 5516 & 622.125 & 2 & -1 \\
\hline Dan Roth & 157 & 4022 & 438.051 & 3 & -1 \\
\hline $\mathrm{Lin} \mathrm{Li}$ & 292 & 4661 & 178.537 & 4 & -4 \\
\hline Jun Wang & 268 & 3872 & 152.947 & 6 & -1 \\
\hline HaoChe & 160 & 2353 & 126.618 & 8 & -5 \\
\hline Eyal Amir & 60 & 1173 & 299.733 & 10 & -2 \\
\hline GiedriusSlivinskas & 60 & 1265 & 158.216 & 11 & -4 \\
\hline Dorothea Wagner & 110 & 1140 & 108.863 & 12 & -3 \\
\hline Marc Joye & 77 & 915 & 101.103 & 13 & -4 \\
\hline Ira Cohen & 57 & 1252 & 262.368 & 10 & -3 \\
\hline
\end{tabular}


TABLE VII. "SCENARIO 3: Position STABLE"

\begin{tabular}{|l|l|l|l|l|l|}
\hline Authors & Publication & Citations & $\begin{array}{l}\text { Average } \\
\boldsymbol{G}_{\text {cit }}\end{array}$ & $\begin{array}{l}\text { H- } \\
\text { index } \\
\text { rank }\end{array}$ & $\begin{array}{l}\text { CGH- } \\
\text { index rank }\end{array}$ \\
\hline $\begin{array}{l}\text { Peter } \\
\text { Druschel }\end{array}$ & 124 & 10185 & 1374.121 & 1 & 1 \\
\hline $\begin{array}{l}\text { Jan Friso } \\
\text { Groote }\end{array}$ & 75 & 1006 & 117.906 & 12 & 12 \\
\hline $\begin{array}{l}\text { Shigang } \\
\text { Chen }\end{array}$ & 45 & 1223 & 320.755 & 13 & 13 \\
\hline S. Keshav & 38 & 805 & 254.263 & 14 & 14 \\
\hline Jian Chen & 83 & 831 & 91.831 & 14 & 14 \\
\hline Yang Yu & 53 & 688 & 111.207 & 15 & 15 \\
\hline Wei Sun & 76 & 588 & 95.210 & 17 & 17 \\
\hline
\end{tabular}

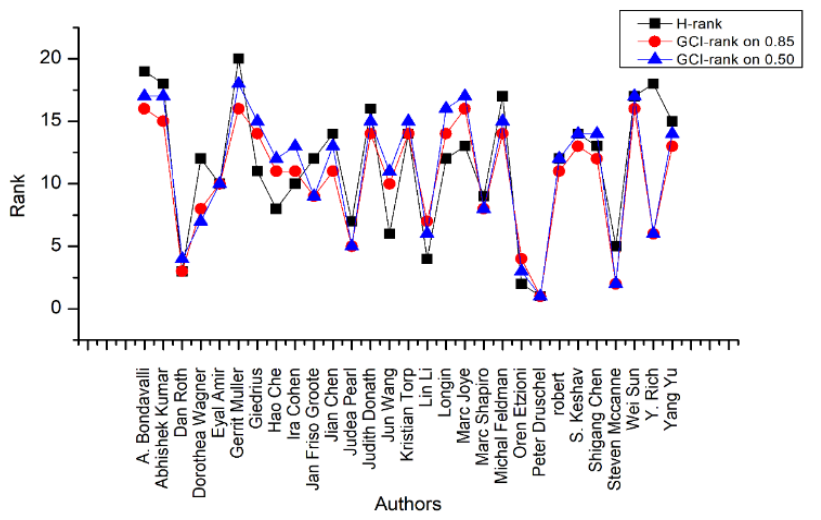

Fig. 5. Comparison of H-Index and CGH with varies damping values.

\section{6) Varied damping factor study}

This is the comparison of the ranks of authors which is clearly depicted in Fig. 5. When the value of the damping factor increases the global citation weight also increases, which ultimately increases the rank of authors.

Authors who have maintained their rank have more influence in the citation network because they got citations from the important nodes in this citation network which excels their weights. We have seen that as the damping factor increase rank of scientist also increases. Results on 0.50 damping factor are very close to the baseline method's result. Authors who have maintained their index with lower damping factor mean they have influence in the citation network.

Fig. 5 depicts the variations of rank with respect to H-rank on varied damping factor. The scholastic approach is adopted to see the variation in rank when considering the impact of global citation weight rather than just counting the citations into the ranking. This variation in rank gives us more insight into the worth of an article. Traditionally, the worth of an article is quantified on the basis of citation count which can give in detail information about the prestige of an article.

\section{CONCLUSIONS AND FUTURE WORK}

Evaluating scientific research production is a challenging task. Authors have proposed GCI which calculates the global citation weight of each publication and index them. It provides more credit to the researchers whose work penetrates many well-known researchers which is quite fair and acceptable quantification. It is concluded that not only the number of citations could affect his/her index but also worth of citing papers is important. Results and discussion show the useful impact of global citations for researcher indexing. It is clear that if citing paper has received more citation then it means it serves more audience and more important for the scientific community. The analysis shows that even if a paper received lesser citations than any other paper but if its citing papers are worthy then it is better.

F-index considers the co-terminal citations into indexing and shows that how the scientific works penetrate into different scientific communities. As a future work, the global citation impact of unique citing authors in addition to simply considering their count can be added in F-Index for improved researcher indexing.

\section{ACKNOWLEDGMENTS}

I am thankful to my respected professor for his support and encouragement. I am also grateful to all my co-authors for their valuable contributions.

\section{REFERENCES}

[1] J. E. Hirsch, "An index to quantify an individual's s scientific research output," Proc. Natl. Acad. Sci. U.S.A., vol. 102, no. 46, pp. 16569 $16572,2005$.

[2] L. Egghe, "Theory and practice of the g -index," Scientometrics, vol. 69, no. 1, pp. 131-152, 2006.

[3] F. J. Cabrerizo, S. Alonso, E. Herrera-Viedma, and F. Herrera, "q 2 Index : Quantitative and qualitative evaluation based on the number and impact of papers in the Hirsch core," vol. 4, pp. 23-28, 2010.

[4] Q. L. Burrell, "On the h-index, the size of the Hirsch core and Jin's Aindex," J. Informetr., 2007.

[5] B. Jin, L. Liang, R. Rousseau, and L. Egghe, "The R-index and ARindices: Complementing the h-index," Chinese Sci. Bull., vol. 52, no. 6, pp. 855-863, 2007.

[6] Q. Wu, "The w-index: A significant improvement of the h-index," J. Am. Soc. Inf. Sci. Technol., vol. 10, no. May, pp. 609-614, 2008.

[7] L. Egghe, "Mathematical theory of the h-index and g-index in case of fractional counting of authorship," J. Am. Soc. Inf. Sci. Technol., 2008.

[8] E. Yan and Y. Ding, "Weighted citation: An indicator of an article's prestige," J. Am. Soc. Inf. Sci. Technol., vol. 61, no. 8, pp. 1635-1643, 2010.

[9] C. T. Zhang, "The e-Index, Complementing the h-Index for Excess Citations," PLoS One, vol. 4, no. 5, 2009.

[10] L. Wildgaard, J. W. Schneider, and B. Larsen, "A review of the characteristics of 108 author - level bibliometric indicators," Scientometrics, vol. 101, no. 1, pp. 125-158, 2014.

[11] M. Ausloos, "A scientometrics law about co-authors and their ranking: The co-author core," Scientometrics, vol. 95, no. 3, pp. 895-909, 2013.

[12] Q. C. Contributions, "edited by Jennifer Sills Biofuels : Clarifying," October, pp. 4-7, 2006.

[13] D. Katsaros, L. Akritidis, and P. Bozanis, "The $f$ index: quantifying the impact of coterminal citations on scientists' ranking," J. Am. Soc. Inf. Sci. Technol., vol. 60, no. 5, pp. 1051-1056, 2009.

[14] A. Daud, R. Abbasi, and F. Muhammad, "Finding rising stars in social networks," in Lecture Notes in Computer Science (including subseries Lecture Notes in Artificial Intelligence and Lecture Notes in Bioinformatics), 2013, vol. 7825 LNCS, no. PART 1, pp. 13-24.

[15] T. Amjad, Y. Ding, A. Daud, J. Xu, and V. Malic, "Topic-based heterogeneous rank," Scientometrics, vol. 104, no. 1, pp. 313-334, 2015.

[16] A. Daud and F. Muhammad, "Consistent Annual Citations based Researcher Index," Collnet J. Sci. Inf. Manag., vol. 8, no. 2, pp. 209216, 2014.

[17] T. Amjad and A. Daud, "Indexing of authors according to their domain 
of expertise," vol. 22, no. 1, pp. 69-82, 2017.

[18] G. Rehman and J. Lee, "Quantifying the Impact of Hot-paper on new Researchers," Proc. 2017 2nd Int. Conf. Commun. Inf. Syst. (ICCIS 2017) , pp. 329-334, 2017.

[19] L. Waltman and V. A. Traag, "Use of the journal impact factor for assessing individual articles need not be wrong," pp. 1-32, 2017.

[20] M. Nykl, K. Ježek, D. Fiala, and M. Dostal, "PageRank variants in the evaluation of citation networks," J. Informetr., vol. 8, no. 3, pp. 683692, 2014.
[21] F. Zhang, "Evaluating journal impact based on weighted citations," Scientometrics, vol. 113, no. 2, pp. 1155-1169, 2017.

[22] U. Senanayake, M. Piraveenan, and A. Zomaya, "The PageRank-index: Going beyond citation counts in quantifying the scientific impact of researchers," PLoS One, vol. 10, no. 8, 2015.

[23] P. Chen, H. Xie, S. Maslov, and S. Redner, "Finding scientific gems with Google's PageRank algorithm," J. Informetr., vol. 1, no. 1, pp. 815, 2007. 